\title{
A Model-less Approach for Estimating Vehicles Sideslip Angle by a Neural Network Concept
}

Bernardo Junqueira Murta ${ }^{1,2}$

bernardo.murta@hds.utc.fr

\author{
Alessandro Correa Victorino ${ }^{2}$ \\ acorreav@hds.utc.fr
}

José Guilherme Coelho Baêta ${ }^{1}$
baeta@demec.ufmg.br

\author{
${ }^{1}$ Mechanical Engineering Department \\ Federal University of Minas Gerais \\ Belo Horizonte, Brazil
}

\author{
${ }^{2}$ Université de Technologie de Compiègne, \\ UMR 7253 CNRS, Heudiasyc \\ CS 60319-60203 Compiègne Cedex
}

literature the use of extended kalman filter (EKF) as state estimators for nonlinear systems. Some authors also use multiple filters, essential to improve the quality of the estimation and to adapt variable driving conditions [2-4].

All those studies were built considering techniques based entirely on physical and dynamic modeling of the vehicle and tires, in addition to techniques based on state observers. These approaches, however, become fragile since there are factors that contribute to the low accuracy of the model used. An example is the load transfer that happens dynamically along the trajectory of vehicles and forces to which they are subjected over time. In this context, some recent studies start from the principle of using machine learning algorithms to assist in the prediction of vehicle dynamic factors that cannot be obtained directly or in a conventional way. In [5] it was used an approach that disregarded tire modeling, where tireground contact forces were estimated through machinelearning algorithms, which lead to additional instrumentation not required. Machine learning algorithms, also, can be used to obtain sideslip angle with maximum error below $5 \%$, when driving in slow speeds [6]. Different approaches were also investigated to determine the slip angle using only the sensors already presented in the vehicle and varying the inputs to the learning system, obtaining good results, but still needing further study about the adherence of the tire with the ground and bank angle[7].

Considering the vehicle lateral dynamics, the study of the tire-ground interaction forces and the sideslip angle is important for the vehicle stability control. The sideslip angle, for instance, determines the displacement direction of the vehicle's center of gravity (CG), which most possibly will not follow the desired trajectory, crucial for the correct work of mobile robots and autonomous vehicles. In this context, many dynamics control systems needs to monitor the sideslip angle in the close loop controller, in order to improve stability [810]. As stated earlier, obtaining these parameters related to vehicle dynamics directly is quite costly and their estimation by model-based methods is inaccurate due to the non-linearity of the system and dynamic variations that are not predicted by these methods. Thus, it is possible to find in the literature algorithms not based on models, which can use readings from a global position system (GPS) [11,12] and by machine learning analysis [13,14]. Moreover, [15] has recently introduced another method, Locally Weighted Projection Regression algorithm (LWPR), to reconstruct tire dynamics, with also high accuracy.

This work aims to evaluate slip angle estimates using neural networks tracking variables with little or no difficulty in obtaining it from the vehicle. In this way, it will be possible to evaluate the possible maneuvers needed for a better training of the network for a higher accuracy of the method. For this, severe driving conditions will be explored, in which it is

This work has granted fundings by CAPES in a doctoral mobility program. This work was supported by LABEX MS2T, ROBOTEX and H2020 European projects ITEAM (675999) and Owheel (872907)

The main author is a PhD candidate at Universidade Federal de Minas Gerais (UFMG) as part of the Graduate Program in Mechanical Engineering

PPGMEC

\begin{abstract}
Over the past few years, vehicle dynamics systems
\end{abstract}
Vehicle control systems, as well as Electronic Stability

In this context, several mathematical models to obtain the forces of tire-ground interaction and sideslip angle are suggested in the literature. The modeling of the system is nonlinear, especially if abrupt maneuvers, in real model, are considered. For this reason, it is widely discussed in the 
expected to reach the limit of vehicle stability and sliding control. With this purpose, the analysis will only be done in a simulation environment for a free variation of the parameters and, thus, validate the proposal.

\section{PROBLEM MODELING}

In this section, it will be discussed the modeling dynamics, which will be used as a base for modeling the neural network in order to get a better prediction of the sideslip angle.

\section{A. Vehicle Modeling}

The vehicle modeling can be simplified by using the bicycle model of the vehicle as showed in Fig. 1. In this model, both front and rear wheels are represented just by one central wheel, vertical movements are ignored, roll is neglected, rear steering angles are null and front steering angles are assumed equals. The simplified bicycle model is represented by the following relationship

$$
\begin{gathered}
\ddot{\Psi}=\left(\frac{1}{I_{z}}\right)\left[l_{f}\left[F_{x 1} \sin \delta+F_{y 1} \cos \delta\right]-l_{r} F_{y 2}\right. \\
\dot{\beta}=\left(1 / m_{v} V_{g}\right)\left[-F_{x 1} \sin (\beta-\delta)+\right. \\
\left.F_{y 1} \cos (\beta-\delta)+F_{y 2} \cos \beta-F_{x 2} \sin \beta\right]-\dot{\psi} \\
\dot{V}_{g}=\left(1 / m_{v}\right)\left[F_{x 1} \cos (\delta-\beta)+F_{y 1} \sin (\delta-\right. \\
\left.\beta)+F_{x 2} \cos \beta-F_{y 2} \sin \beta\right]
\end{gathered}
$$

where $\psi$ is the true heading of the vehicle, $I_{z}$ the yaw moment of inertia, $F_{x, y, 1,2}$ are the longitudinal and lateral forces of front and rear wheels, $m_{V}$ is the vehicle's mass, $V_{g}$ is the total velocity at the center of gravity, $\delta$ is the steering angle $\beta$ is the sideslip angle, $l_{f}$ and $l_{r}$ are the distances of the front and rear axles, respectively, to the $\mathrm{CG}$.

The sideslip angle, besides being able to be obtained by a direct integration of (2), it is defined as the angle between vehicle's longitudinal speed and actual speed, considering the vehicle's body frame placed at the CG.

$$
\beta=\arctan \left(V_{y} / V_{x}\right)
$$

Since the sideslip angle should be small for control, it can be considered a standard $\beta \ll 1$ and then $V_{y}=\beta V_{x}$. By deviating (4), then, it can be assumed that, for constant longitudinal speed

$$
\dot{\beta}=a_{y} / V_{x}-\dot{\psi}
$$

where $\dot{\psi}$ is the true heading of the vehicle. Equations (2), (4) and (5), then, indicate the sideslip angle relationship, which can be assumed as input variables for a prediction algorithm.

\section{B. Neural Network modeling}

Neural networks $(\mathrm{NN})$, one machine learning method, are model-less approximators, which means that they are capable of perform well without any prior information from the system's modeling. Moreover, the method can be done either by relating known examples or without any knowledge about the outputs during training. Thus, these methods can become

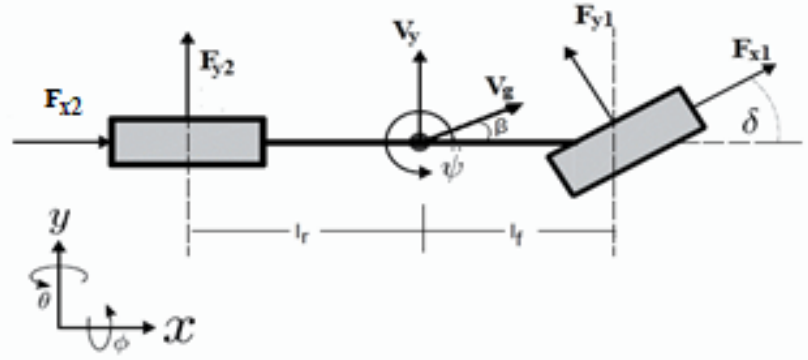

Fig. 1. Bicycle Model

a powerful tool for obtaining parameters related to the vehicle dynamics that would be difficult and economically unviable to obtain through dedicated sensors [16]. The basic description of this algorithm is

$$
Y_{k}=f\left(U_{k}, Z_{k}\right)
$$

where $Y_{k}$ is the output of the network, $U_{k}$ are the inputs and $Z_{k}$ are the systems parameters, and the simple structure that forms the network are called neurons [17]. Neurons compose different layers, named input layer, hidden layers, and output layer. Between each layer, a series of simple operations are performed,

$$
S_{j}=\sum a_{j} w_{i j}+b_{j}
$$

where $S_{j}$ represents the output from a neuron, $w_{i j}$ are the weights trained that each neuron describe the influence of the input from the previous layer. The sum is biased by a factor $b_{j}$. Every node on each layer is then activated by an activation function and the result is the output for that particular node, which is then passed as part of the inputs to the nodes of the next layer, until the output layer is finally reached. This process is known as forward propagation.

Upon reaching the output layer, the network produces a value which is different from the target's value. The error is then propagated back into the network, updating the weights on each neuron in order to reduce the loss function [16]. This process is known as backpropagation. The learning process continues in a set of epochs until an acceptable error is reached, and the number of epochs depends on convergence parameters.

\section{SIMULATIONS}

To simulate the operating conditions of any vehicle and to obtain the variables of interest using virtual sensors, it was used the IPG Carmaker ${ }^{\circledR}$ software and the sideslip angle was defined as the target parameter to be studied. The input variables to describe the NN structure follows the parameters shown in (2), (4) and (5), and a total of 5 inputs were chosen (steering angle $\delta$, longitudinal velocity $V_{x}$, lateral acceleration $a_{y}$, the yaw rate $\psi$ and the friction coefficient $\mu$ ). Regarding all input variables, most of them can be accessed by low-cost sensors and some are already presented in most cars. The friction coefficient is the most difficult to be 


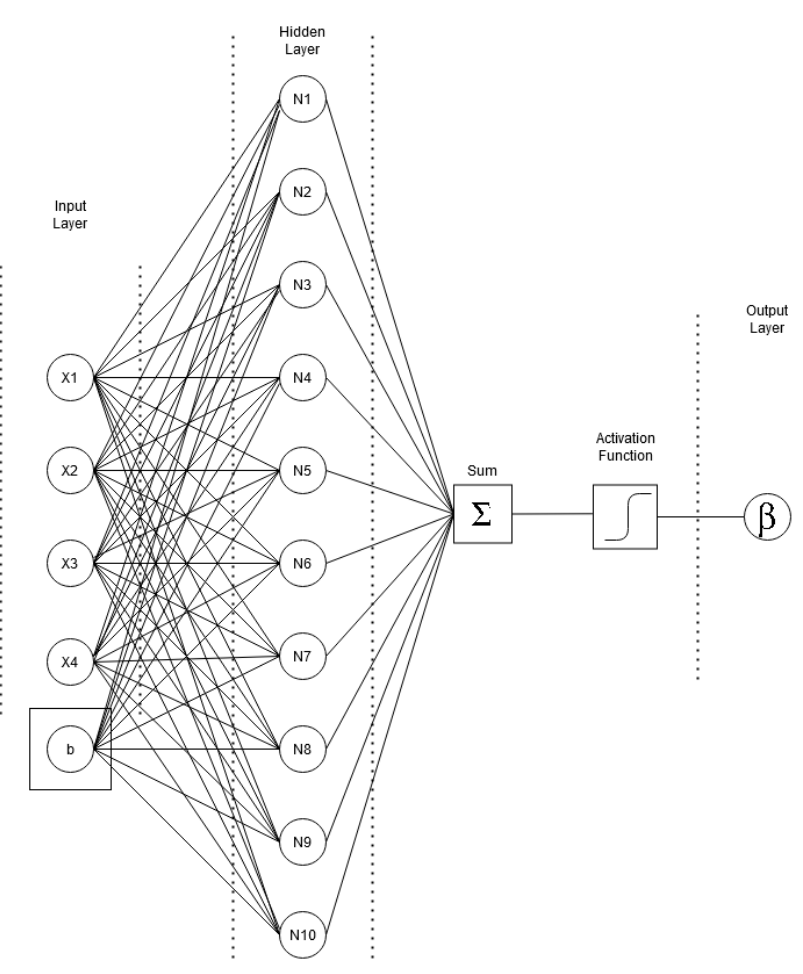

Fig. 2. Neural Network structure and inputs

accessed, but crucial to slip dynamics that affect tire-ground forces, being a parameter that needs to be predicted. Therefore, several works studied the estimation of the friction coefficient in order to improve stability control, considering even vehicle and tire modeling, image processing and machine learning techniques (neural networks, fuzzy logic) [17-21]. Despite being a basic analytical tool, fuzzy systems have shown to deal well with dynamic parameters uncertainties when good prerequisites are put in place for the application, and by using cascade approaches [22-23].

For the NN training, it was used a Matlab ${ }^{\circledR}$ toolbox, due to its simplicity in use, and a classic feedforward NN structure of 5-10-1 neurons was used, as shown in Fig 2. For training, test and validation, it was used a 70/15/15 split which experienced best training performance, along with a sigmoid activation function. Moreover, a backpropagation LevenbergMarquardt algorithm was chosen to minimize training errors.

\section{A. Training Datasets}

Most works presented in the literature uses many different tests during the training phase to build the neural network. This procedure seems correct since the network needs data that covers most part of the intended range of the study. On the other hand, when applying into a real-world experiment it might add risks to the drivers end experimental researchers, since most of the tests are performed close to the vehicle's slip limit. Thus, it is necessary to create a test and simulation protocol to guarantee the integrity of the conductor during the test and to minimize operational and computational costs with a large dataset.

In this context, the present work used only simulations in which the test parameters can be freely varied, and so that the size of the dataset was not too extensive. For this, 3 different tests were defined a priori whose lateral excitation can reach the slip limit: Slalom 18m, Slalom 36m, Sine with Dwell. In the IPG Carmaker ${ }^{\circledR}$ software, it was chosen a Volkswagen Beetle for the tests, with a $70 \mathrm{~kg}$ load added at the drivers'

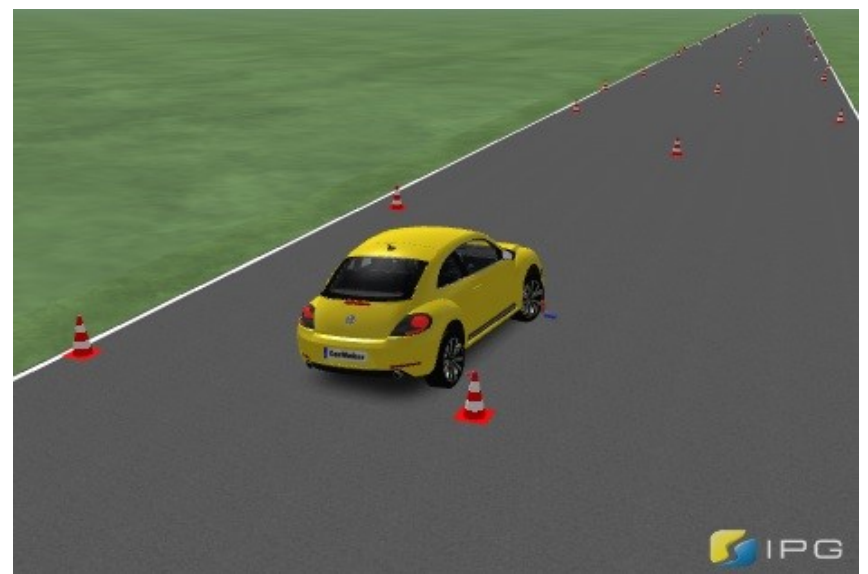

Fig. 3. Software environment during a slalom test (IPGMovie)

TABLE I. TRAIN, TEST AND VALIDATION ACCURACY FOR TRAINING PHASE - SECTION I

\begin{tabular}{|l|c|c|c|c|}
\hline \multirow{2}{*}{$\begin{array}{c}\text { Train } \\
\text { Phase }\end{array}$} & \multicolumn{2}{|c|}{ NN1 } & \multicolumn{2}{c|}{ NN2 } \\
\cline { 2 - 5 } & $\boldsymbol{M S E}$ & $\boldsymbol{R}$ & $\boldsymbol{M S E}$ & $\boldsymbol{R}$ \\
\hline Train & $8.00 \mathrm{E}-3$ & $9.70 \mathrm{E}-1$ & $1.05 \mathrm{E}-1$ & $8.03 \mathrm{E}-1$ \\
\hline Test & $6.51 \mathrm{E}-3$ & $9.79 \mathrm{E}-1$ & $8.80 \mathrm{E}-2$ & $8.30 \mathrm{E}-1$ \\
\hline Valid. & $5.73 \mathrm{E}-3$ & $9.76 \mathrm{E}-1$ & $1.04 \mathrm{E}-1$ & $7.97 \mathrm{E}-1$ \\
\hline
\end{tabular}

position. Fig 3 shows the software environment when performing a slalom test.

For the running simulations, in order to get a wide range of operation conditions, tests were run varying the longitudinal speed from $60-110 \mathrm{~km} / \mathrm{h}$ and friction coefficient of $0.6,0.8$ and 1.0. In this way, the actual maneuver is only performed after reaching constant speed. With the test's simulation and acquisition of variables of interest, data was separated in two different datasets. NN1 contains data from both slalom tests; and NN2 contains all data, adding the sine with dwell test. This procedure aims to indicate if a neural network will produce better results when adding different datasets to the training process. For creating each dataset, it was avoided stationary results so that the training data fully represents the maneuvers performed. The training, test and validation accuracy for both datasets are presented in Tab. 1. The training results were then applied to a different test, which consists of two lane changes, for accuracy analysis.

Despite having similar characteristics regarding steer angles range, adding the sine with dwell data to the training process affected negatively to the training accuracy. Likewise, when applying the NN2 training results to a different dataset, it did not result in a significant improvement in the prediction of the vehicle's sideslip angle, as seen in Fig. 4 and Fig. 5 attesting that this behavior continued to occur for different test's speeds. Thus, the data corresponding to the "sine with dwell" test were disregarded for validation.

\section{B. Validation}

For the validation stage, the same procedure was conducted in order to compare the inclusion of different dataset to the training phase. However, the comparison considered both slalom tests and the addition of a double-lane change test. The two datasets will now be related as NN1 and NN3. For the NN3 dataset it was also included acceleration and steady speed stages in order to reduce base sideslip offset, as seen in Fig.4 and Fig. 5. 

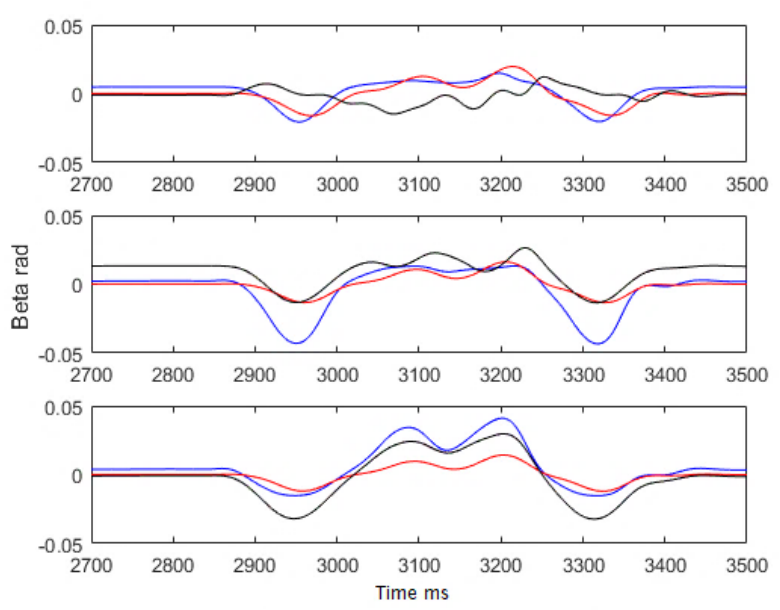

- NN1 - Reference- - NN2

Fig. 4. Comparison between sideslip prediction results for different training datasets NN1, NN2 and Simulated, for longitudinal speed of $80 \mathrm{~km} / \mathrm{h}$ and friction coefficient varying from $0.6,0.8$ and 1 , respectively, in a lane change environment.
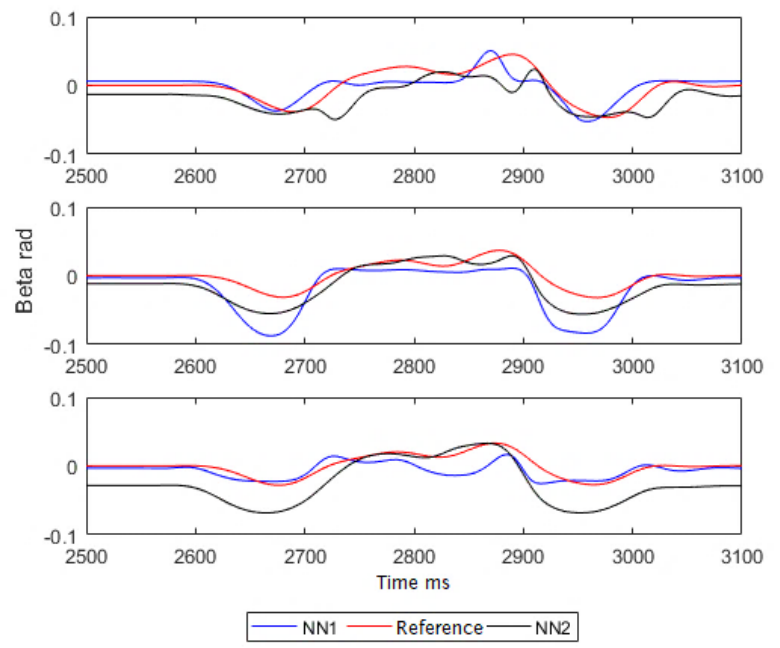

Fig. 5. Comparison between sideslip prediction results for different training datasets NN1, NN2 and Simulated, for longitudinal speed of $100 \mathrm{~km} / \mathrm{h}$ and friction coefficient varying from $0.6,0.8$ and 1 , respectively, in a lane change environment.

The training, test and validation accuracy for both datasets are presented in Tab. 2. The training results were then applied to a different test, which consists of an s-shaped road with vehicles moving, for accuracy analysis, as seen in Fig. 6. In this test, depending on the vehicle speed, instances of lane change are different since the moving objects have constant speeds for all tests.

The dataset created for NN3 resulted in lower errors compared to NN1, as seen in Tab. 3, but mostly related to the acceleration and steady speed phases added. Nonetheless, when applying both networks to the validation test, the addition of the lane change dataset to the training phase was able to produce better sideslip predictions, comparing to the results obtained from the simulation itself. This behavior can be found in all tests, regardless of the longitudinal speed and the friction coefficient of the road, as seen in Fig. 7 and Fig. 8 .

In all tests at $80 \mathrm{~km} / \mathrm{h}$, it can be observed in Fig. 7 a deviation of the predicted values for slip angle that occurred
TABLE II. TRAIN, TEST AND VALIDATION ACCURACY FOR TRAINING PHASE - SECTION II

\begin{tabular}{|l|c|c|c|c|}
\hline \multirow{2}{*}{$\begin{array}{c}\text { Train } \\
\text { Phase }\end{array}$} & \multicolumn{2}{|c|}{ NN1 } & \multicolumn{2}{c|}{ NN3 } \\
\cline { 2 - 5 } & $\boldsymbol{M S E}$ & $\boldsymbol{R}$ & $\boldsymbol{M S E}$ & $\boldsymbol{R}$ \\
\hline Train & $8.00 \mathrm{E}-3$ & $9.70 \mathrm{E}-1$ & $3.13 \mathrm{E}-3$ & $9.77 \mathrm{E}-1$ \\
\hline Test & $6.51 \mathrm{E}-3$ & $9.79 \mathrm{E}-1$ & $4.89 \mathrm{E}-3$ & $9.75 \mathrm{E}-1$ \\
\hline Valid. & $5.73 \mathrm{E}-3$ & $9.76 \mathrm{E}-1$ & $3.37 \mathrm{E}-3$ & $9.65 \mathrm{E}-1$ \\
\hline
\end{tabular}

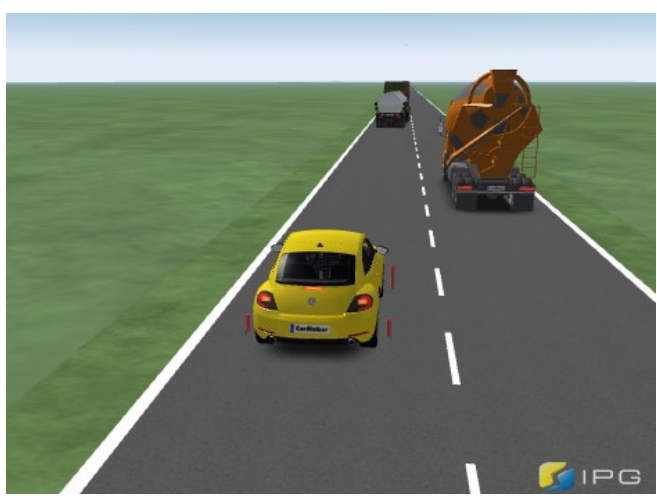

Fig. 6. Software environment when performing various maneuvers with moving objects on the road (IPGMovie)

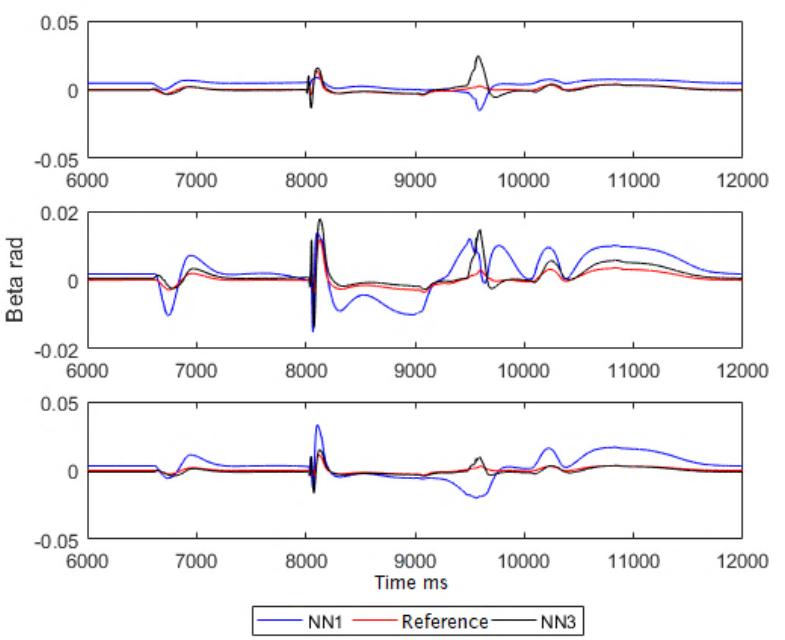

Fig. 7. Comparison between sideslip prediction results for different training datasets NN1, NN3 and Simulated, for longitudinal speed of $80 \mathrm{~km} / \mathrm{h}$ and friction coefficient varying from $0.6,0.8$ and 1 , respectively, when performing various maneuvers.

at $9600 \mathrm{~ms}$, approximately. During the simulation procedure, at this moment, the vehicle performed a lane change maneuver while entering in a curve, which explains the increase in the sideslip angle for low friction coefficient. Such maneuvers were not present in the training's datasets, which comes to a fact that the current network proves to be quite dependent on the training data. In such cases, larger datasets are needed so that most or all operating conditions are covered. However, the dataset size also plays an important role on the time spent and computational resources for training.

To get a better understanding of the event error by the NN model and to properly address, a simple track (Fig. 9) was chosen containing $90^{\circ}$ turns with different radius which, so far, was not included in the training datasets. The simulation results for the sideslip angle were then compared with the NN3 training results, presented in Fig. 10, for two different friction coefficient and constant speed. 

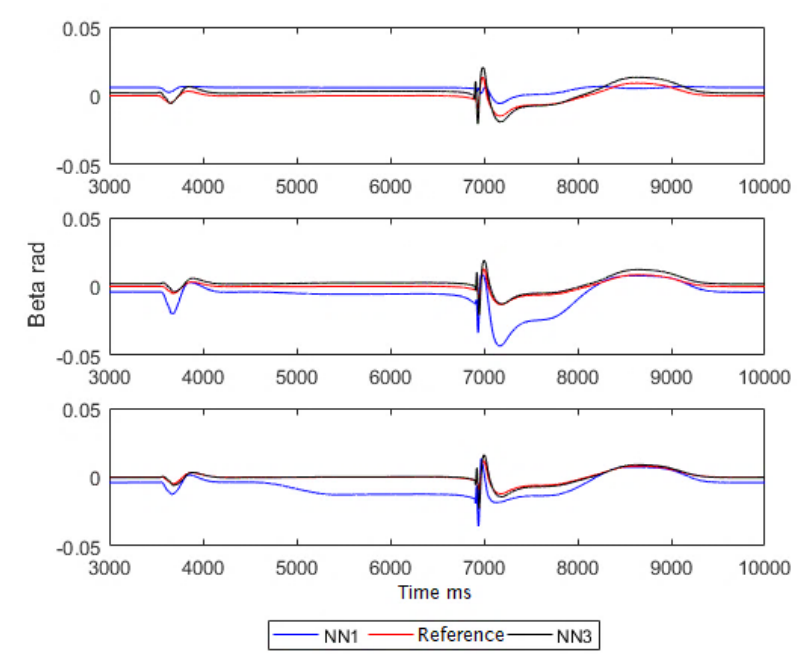

Fig. 8. Comparison between sideslip prediction results for different training datasets NN1, NN3 and Simulated, for longitudinal speed of $100 \mathrm{~km} / \mathrm{h}$ and friction coefficient varying from $0.6,0.8$ and 1 , respectively, when performing various maneuvers.

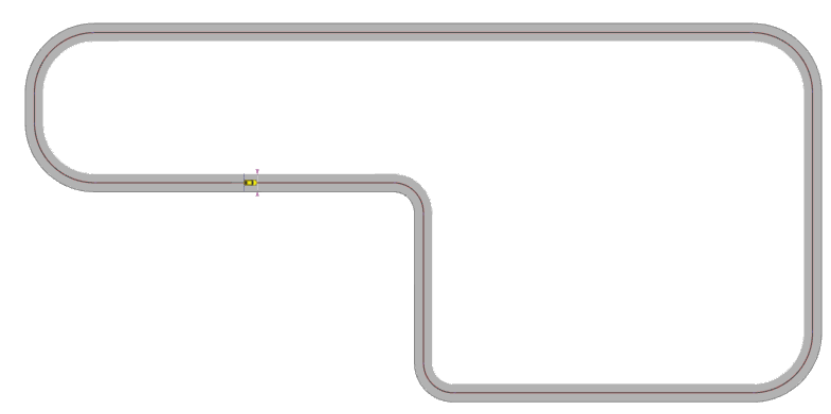

Fig. 9. Simple track simulation with cornering turns

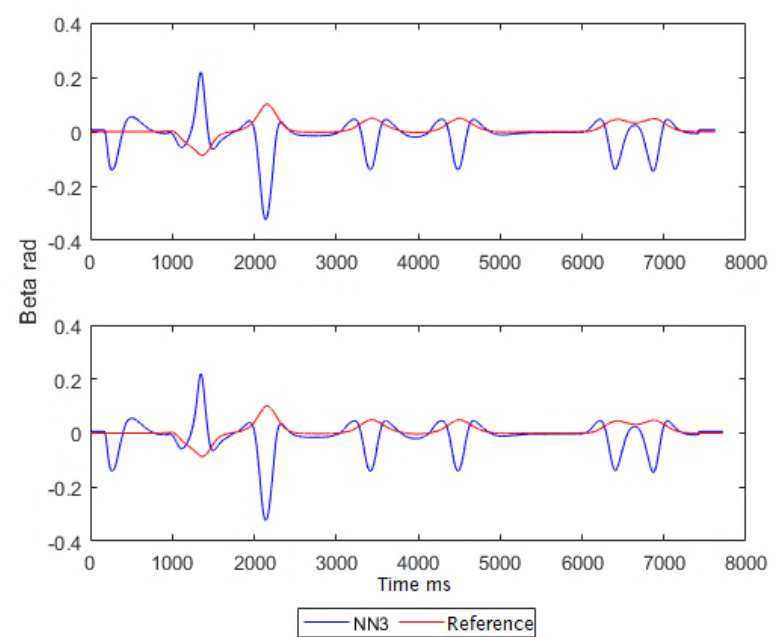

Fig. 10. NN model errors when predicting a non-trained event. Simulation results for longitudinal speed $80 \mathrm{~km} / \mathrm{h}$ and friction coefficient varying from 0.6 and 1 , respectively, when performing various $90^{\circ}$ turns

The results shown in Fig. 10 contributes to the previous statement, in which the current NN model is mostly dependent on the training data, corresponding to a limitation of the method. Extrapolating to real cases, it becomes a very difficult task to obtain experimental data on most of the vehicle's operating conditions, many of which pose risks to the test driver and researchers. Furthermore, when analyzing the data distribution from what was used in Fig. 7, despite having a
TABLE III. MEAN SQUARE ERRORS FOR NN1 AND NN3 WHEN APPLYING TO A RANDOMIC TEST

\begin{tabular}{|c|c|c|c|c|c|c|}
\hline \multirow{2}{*}{$\boldsymbol{\mu}$} & \multicolumn{3}{|c|}{ NN1 } & \multicolumn{3}{c|}{ NN3 } \\
\cline { 2 - 7 } & $\mathbf{6 0} \mathbf{k m} / \mathbf{h}$ & $\mathbf{8 0} \mathbf{k m} / \boldsymbol{h}$ & $\begin{array}{c}\mathbf{1 0 0} \mathbf{k m} / \\
\boldsymbol{h}\end{array}$ & $\mathbf{6 0 k m} / \mathbf{h}$ & $\mathbf{8 0 k m} / \boldsymbol{h}$ & $\begin{array}{c}\mathbf{1 0 0} \mathbf{k m} / \\
\boldsymbol{h}\end{array}$ \\
\hline 1.0 & $3.97 \mathrm{e}-4$ & $3.72 \mathrm{e}-4$ & $4.67 \mathrm{e}-4$ & $3.16 \mathrm{e}-5$ & $3.92 \mathrm{e}-5$ & $4.76 \mathrm{e}-5$ \\
\hline 0.8 & $1.45 \mathrm{e}-4$ & $7.05 \mathrm{e}-5$ & $1.29 \mathrm{e}-4$ & $4.93 \mathrm{e}-6$ & $6.41 \mathrm{e}-6$ & $1.07 \mathrm{e}-5$ \\
\hline 0.6 & $1.10 \mathrm{e}-4$ & $1.50 \mathrm{e}-4$ & $1.88 \mathrm{e}-4$ & $5.49 \mathrm{e}-5$ & $6.05 \mathrm{e}-5$ & $7.76 \mathrm{e}-5$ \\
\hline
\end{tabular}

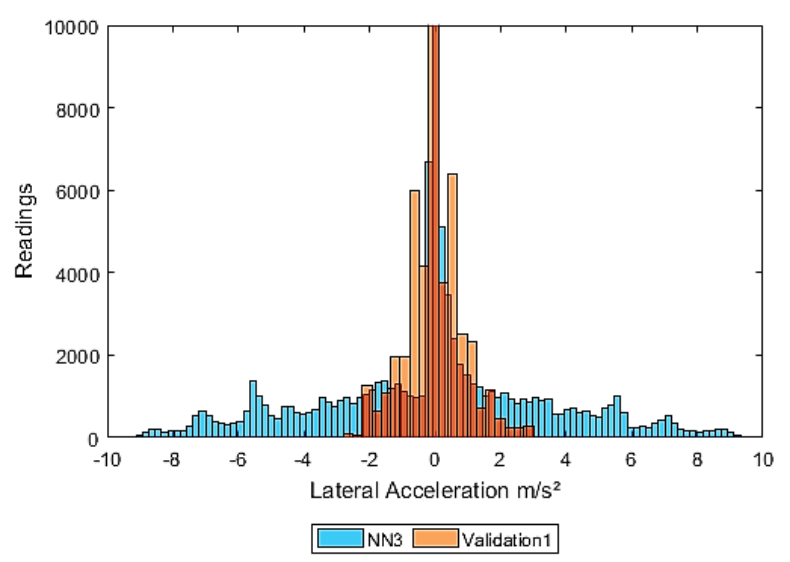

Fig. 11. Summary of instances for Lateral Acceleration of NN3 and the simulation of various maneuvers with moving objects on the road.

restricted range of operation conditions, the fact that validation dataset had more centered data it turned out that the NN3 training dataset was insufficient to represent the data entirely, making it necessary to add more data to the training phase. This difference can be seen in Fig. 11, for lateral acceleration data.

However, results obtained by proper training have shown to be promising, even in most nonlinear regions, of larger sideslip angles. For a purely neural network model, the presented behavior of the neural network output for untrained events corroborates to the need of creating a set of standard tests to access most operating conditions to be faced by the vehicle. In this way, such neural networks models can improve stability control by obtaining better predictions for the vehicle's lateral dynamics without the need of additional instrumentation.

\section{CONCLUSIONS}

The present work aimed to model vehicle parameters using neural networks to access information regarding the lateral dynamics of the vehicle, i.e. the sideslip angle. This variable information is important for obtaining a better stability control and increase in safety. Also, when applying to mobile robots and autonomous vehicles, it can increase the accuracy of the maneuver predetermined and motion planning.

The tests analysis for testing neural networks behavior give knowledge of which maneuvers are important to be addressed and studied. Regarding the results obtained, the dataset which included slalom tests and double lane changing information performed better for predicting the sideslip angle, when applying for low steering angles. These results were achieved after a training accuracy of more than $97 \%$ obtained for both datasets, and errors lower than $1 \%$.

When applying the model to a non-trained data, with higher steering angles, when performing a low radius curve, the results were very discrepant, as stated in Fig. 7. Such 
behavior shows that the trained neural network is quite dependent on the dataset inserted during the training phase of the network and, with any data not previously trained, it will not perform well, with large prediction errors. However, adding a lot of data to the network increases its complexity and the need for a greater computational processing during the training phase. Still, a lot of data necessary to be included in the network may not be very feasible to obtain outside of controlled environments, such as simulation software, and very sudden maneuvers can bring risks to drivers during the acquisition of data, many of them in the limit of stability of the vehicle.

This model behavior should be addressed in the following works, also including other maneuvers and adding bank angle and road grade information. Therefore, with the knowledge obtained by these analyses it can be possible to create a protocol for standardized tests procedure during the training phase.

Other important assumption to be made for future works is combining model-based algorithms with neural networks prediction methods in order to create a hybrid model. In this way, vehicles dynamics parameters can be accessed by modelbased procedure and non-linearities overcame by training methods.

\section{ACKNOWLEDGMENT}

This study was financed in part by the Cooperação de Aperfeiçoamento de Pessoal de Nível Superior - Brazil (CAPES) - Finance code 001. The authors acknowledge CAPES for the funding by means of the exchange program CAPES PrInt that made this work possible and the international agreement between Federal University of Minas Gerais (UFMG) and University of Technology of Compiègne (UTC).

\section{REFERENCES}

[1] Doumiati M, Charara A, Victorino A, Lechner D, 2012. Vehicle dynamics estimation using Kalman filtering. doi: 10.1002/ 9781118578988

[2] Cordeiro, R.A., Ribeiro, A.M., Azinheira, J.R., Victorino, A.C., Ferreira, P.A.V., De Paiva, E.C., Bueno, S.S., 2017. Road grades and tire forces estimation using two-stage extended Kalman filter in a delayed interconnected cascade structure. IEEE Intell. Veh. Symp. Proc. 115-120. https://doi.org/10.1109/IVS.2017.7995707

[3] Hong, S., Lee, C., Borrelli, F., Hedrick, J.K., 2015. A novel approach for vehicle inertial parameter identification using a dual kalman filter. IEEE Trans. Intell. Transp. Syst. 16, 151-161. https://doi.org/10.1109/TITS.2014.2329305

[4] Jin, X., Yin, G., 2015. Estimation of lateral tire-road forces and sideslip angle for electric vehicles using interacting multiple model filter

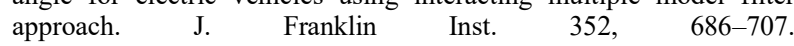
https://doi.org/10.1016/j.jfranklin.2014.05.008

[5] Acosta, M., Alatorre, A., Kanarachos, S., Victorino, A., Charara, A., 2017. Estimation of tire forces, road grade, and road bank angle using tire model-less approaches and Fuzzy Logic. IFAC-PapersOnLine 50, 14836-14842. https://doi.org/10.1016/j.ifacol.2017.08.2583

[6] Sasaki, H. and Nishimaki, T., "A Side-Slip Angle Estimation Using Neural Network for a Wheeled Vehicle," SAE Technical Paper 200001-0695, 2000, https://doi.org/10.4271/2000-01-0695.

[7] Melzi, S., Sabbioni, E. ,2011. On the vehicle sideslip angle estimation through neural networks: Numerical and experimental results. Mechanical Systems And Signal Processing, 25(6), 2005-2019. doi: 10.1016/j.ymssp.2010.10.015

[8] A. Alatorre, A. Victorino, A. Charara Sideslip estimation algorithm comparison between Euler angles and quaternion approaches with black box vehicle model, IEEE 15th International Workshop on Advanced Motion Control (AMC 2018). Tokyo, Japan 2018.

[9] Tchamna, R. and Youn, I. Yaw rate and side-slip control considering vehicle longitudinal dynamics Int.J Automot. Technol. (2013) 14:53. https://doi.org/10.1007/s12239-013-0007-1.

[10] M. Abe, Y. Kano, K. Suzuki, Y. Shibahata and Yoshimi Furukawa. Side-slip control to stabilize vehicle lateral motion by direct yaw moment. JSAE Review, Volume 22, Issue 4, 2001, Pages 413-419, ISSN 0389-4304, https://doi.org/10.1016/S0389-4304(01)00124-2.

[11] F. Farroni, N. Pasquino, E. Rocca, and F. Timpone, "A comparison among different methods to estimate vehicle sideslip angle," in Proceedings of the World Congress on Engineering, vol. 2, 2015.

[12] D. Piyabongkarn, R. Rajamani, J. Grogg, and J. Lew, "Development and experimental evaluation of a slip angle estimator for vehicle stability control," IEEE Transactions on Control Systems Technology, vol. 17, no. 1, pp. 78-88, 2009.

[13] Busnelli, F., Panzani, G., Corno, M., and Savaresi, S. M. (2017). Twowheeled vehicles black-box sideslip angle estimation. In 2017 IEEE 56th Annual Conference on Decision and Control (CDC), pages 351356. IEEE.

[14] De Martino, M., Farroni, F., Pasquino, N., Sakhnevych, A., and Timpone, F. (2017). Real-time estimation of the vehicle sideslip angle through regression based on principal component analysis and neural networks. In 2017 IEEE International Systems Engineering Symposium (ISSE), pages 1-6. IEEE.

[15] K. Iyer, B. Shyrokau and V. Ivanov, "Offline and Online Tyre Model Reconstruction by Locally Weighted Projection Regression," 2020 IEEE 16th International Workshop on Advanced Motion Control (AMC), Kristiansand, Norway, 2020, pp. 311-316, doi: 10.1109/AMC44022.2020.9244310.

[16] Belic I, 2012. Neural Networks and static modelling. Neural Netw Static Model Recurr Neural Netw Soft Comput Dr. Mahmoud ElHefnawi

[17] Q. Cheng, A. Correa-Victorino and A. Charara, "A new nonlinear observer using unscented Kalman filter to estimate sideslip angle, lateral tire road forces and tire road friction coefficient," 2011 IEEE Intelligent Vehicles Symposium (IV), Baden-Baden, 2011, pp. 709714, doi: 10.1109/IVS.2011.5940501.

[18] Pasterkamp, W. and Pacejka, H. (1997). Application of neural networks in the estimation of tire/road friction using the tire as sensor. Sae Technical Papers.

[19] Ribeiro, A.M., Moutinho, A., Fioravanti, A.R. et al. Estimation of tireroad friction for road vehicles: a time delay neural network approach. J Braz. Soc. Mech. Sci. Eng. 42, 4 (2020). https://doi.org/10.1007/s40430-019-2079-y

[20] Šabanovič, E.; Žuraulis, V.; Prentkovskis, O.; Skrickij, V. Identification of Road-Surface Type Using Deep Neural Networks for Friction Coefficient Estimation. Sensors 2020, 20, 612.

[21] Kanwar Bharat Singh \& Saied Taheri (2015) Estimation of tire-road friction coefficient and its application in chassis control systems, Systems Science \& Control Engineering, 3:1, 39-61, DOI: $10.1080 / 21642583.2014 .985804$

[22] Valentin Ivanov, Barys Shyrokau, Klaus Augsburg, Vladimir Algin, Fuzzy evaluation of tyre-surface interaction parameters, Journal of Terramechanics, Volume 47, Issue 2, 2010, Pages 113-130, ISSN 0022-4898, https://doi.org/10.1016/j.jterra.2009.08.003.

[23] Ivanov, Valentin, et al. "Advancement of vehicle dynamics control with monitoring the tire rolling environment." SAE International Journal of Passenger Cars-Mechanical Systems 3.2010-01-0108 (2010): 199-216. 